\title{
The stability of laminar symmetric separated wakes
}

\author{
By IAN P. CASTRO \\ School of Engineering Sciences, University of Southampton, Highfield, Southampton, SO17 1BJ, UK
}

(Received 6 October 2003 and in revised form 20 October 2004)

Time-dependent computations of the two-dimensional incompressible uniformvelocity laminar flow past a normal flat plate (of unit half-width) in a channel are presented. Attention is restricted to cases in which the well-known anti-symmetric (von Kármán-type) vortex shedding is suppressed by the imposition of a symmetry plane on the downstream plate centreline. With a further symmetry plane at the channel's upper boundary, the only two governing parameters in the problem are the channel half-width, $H$, and the Reynolds number, $R e$ (based on the body half-width and the upstream velocity, $U$ ). The former is restricted to the range $3 \leqslant H \leqslant 30$ and the interest lies in determining the nature of the initial instability which occurs in the separated wake as $R e$ is gradually increased. It is found that for sufficiently large $H$ and at a critical $R e$, a long-time-scale global (supercritical) instability is initiated, which in its saturated (limit) state takes the form of 'lumps' of vorticity being periodically shed from the tail end of the separated bubble. Stability calculations of corresponding mean flow profiles (typical of those found in the separated wake) are undertaken by examining the impulse response of particular profiles via appropriate solution of the Orr-Sommerfeld equation. The results of this analysis extend those available from related published work and are consistent with the behaviour found from the numerical computations. Taken together, all the results suggest that this type of global instability may be generic to many kinds of separated wakes and, indeed, may provide the fundamental explanation for the very low-frequency oscillations often noticed in fully turbulent wake bubbles.

\section{Introduction}

An interesting class of laminar flow over a two-dimensional bluff body is that in which the (Kármán-type, asymmetric, periodic) vortex shedding is prevented by the imposition of symmetry on the wake centreline, either by the physical addition of a central splitter plate or, analytically or numerically, by simple enforcement of a frictionless boundary condition. After numerous attempts by many authors over many years the asymptotic nature of such flows - i.e. the form of the wake as $R e \rightarrow \infty-$ was finally revealed by Chernyshenko (1988). Throughout this paper $R e$ is based on the (unit) half-width of the body and the velocity, $U$, far upstream. The problem becomes a little more realistic if the body is just one of an infinite cascade of identical bodies, being located at the centre of a plane channel (as in the case of a body in a wind tunnel, for example). This imposes a further parameter, defined here as $H$, the ratio of the channel width to the body width. Numerical solutions for circular cylinder cascades have been presented by Fornberg (1991) (with $R e \leqslant 400$ in the present terms and $5 \leqslant H \leqslant 100$ ) and, very recently and to much higher Reynolds numbers, by Gajjar \& 
Azzam (2004). Similar computations for flat-plate cascades have been by presented by Natarajan, Fornberg \& Acrivos (1993) (with $R e \leqslant 400$ and $5 \leqslant H \leqslant 25$ ) and Ingham, Tang \& Morton (1990) (with $R e \leqslant 500$ and $H=2$ ). Chernyshenko's (1988) asymptotic theory was extended to the cascade case by Chernyshenko \& Castro (1993) and numerical computations gave results which were shown to tend qualitatively towards those expected, but only at the very highest Reynolds numbers. The additional effects of density stratification have also been considered (Chernyshenko \& Castro 1996).

We have shown recently, using further numerical computations, that although there are many features of the wake which do indeed have the form predicted by the general asymptotic solutions, there are nonetheless some features which do not convincingly conform with the Chernyshenko theory (Castro 2002). Quantitative agreement with the asymptotic 'long eddy' solution (for which the theory requires $H / R e \rightarrow 0, H \rightarrow \infty$ in the unstratified case) does not occur, for example. It was also found that the very different, boundary-layer-type solutions which appear at low $H$ (i.e. $R e \rightarrow \infty, H=O(1)$ ) become very difficult to obtain once $R e$ exceeded about 250 and since some initial time-dependent computations indicated that periodic solutions appear - for $R e>180$ in the case of $H=10$, for example; the steady solutions which could sometimes be obtained at higher Re were clearly unstable. This important issue was not pursued at the time although it is (arguably) obvious intuitively that the highReynolds-number flows are likely to be inherently unstable. Note that Smith (1985) has also discussed the boundary-layer-type solutions which appear for $H=O(1)$, and numerical solution of the appropriate boundary-layer equations (as opposed to the full Navier-Stokes (NS) equations) is relatively straightforward. (Whether or not steady solutions of the NS equations can be obtained for such cases depends on the characteristics of the numerical scheme.)

Linear stability theory has been very successful in revealing many crucial features of the initial instabilities in parallel shear flows. Wake flows with uniformly positive velocities have been studied (e.g. Papageorgiou \& Smith 1989) but, in particular, a number of authors have studied the stability of general velocity profiles which contain reversed flow regions. For example, for a mixing layer profile defined by

$$
u=1+R \tanh \left(\frac{1}{2} y\right),
$$

where the velocity, $u$, is normalized by the average velocity of the two streams and $R$ is the ratio of the difference in these two velocities to their sum, Huerre \& Monkewitz (1985) showed that the inviscid flow becomes absolutely unstable if $R>1.315$. This corresponds to cases in which the magnitude of the backflow velocity exceeds that of the forward flowing stream by more than about $13.6 \%$. Convective instability occurs for $0.84<R<1.315$ so, for increasing $R$, starts without any backflow at all. Later studies have considered similar, but viscous cases. Perhaps more relevant to the present study (but see later) is the work of Hultgren \& Aggarwal (1987), who considered the rather different profile defined by

$$
u=1-\lambda \exp \left(-y^{2} \ln (2)\right),
$$

where the velocity is normalized by its maximum value, $U$ say, $y$ is normalized by the wake's half-width and $\lambda$ is a measure of the amount of reverse flow. Their emphasis was on the modes corresponding to Kármán-type shedding. However, they also found that for (what they called) anti-symmetric modes - when symmetry is enforced on the wake centreline $(y=0)$ so the flow is forced to be more like a mixing layer - convective instability begins when $R e=53$. Here, $R e$ is based on the wake half-width and the maximum velocity difference, $\lambda U$. Note that in these terms, the critical $R e$ does not depend on $\lambda$. They did not report the $R e$ required for the onset of absolute instability. 
Flow type

Mixing layer (equation (1.1))

Mixing layer (equation (1.1)) with constraining lower boundary

Modified Falkner-Skan profiles (boundary layer with reverse flow)

Unrestricted wake (equation (1.2) but with symmetry at $y=0$ )

Wakes (various profiles) with imposed central symmetry and upper slip boundary
Reference

Huerre \& Monkewitz

(1985), inviscid

Hammond \& Redekopp

(1998, appendix), viscous

(but high $R e$ )

Hammond \& Redekopp

(1998), viscous (but

high $R e$ )

Hultgren \& Aggarwal

(1987), viscous

Present work, viscous
Salient result

Absolutely unstable for

$U_{m}<-0.136(R>1.315)$

More backflow (than above) needed for absolute instability

Absolutely unstable for $U_{m}<-0.3$

Convective instability at $R e>53$ (independent of $U_{m}$ ). No study of conditions for absolute instability.

Convective and absolute instabilies depending on $H$ and also (for the latter) $R e$, see $\S 4$.

TABLE 1. Summary of flow types, corresponding references and salient results.

$U_{m}$ is the minimum backflow velocity.

These cases refer to free shear layers, remote from walls. A practically very important class of cases occurs when there is a wall present - usually on the low (negative) velocity side of the shear layer. One then expects the stability characteristics of the free shear layer, arising from the inflection point in the velocity profile, to be modified. The effects of the wall on the convective and absolute instability characteristics have been studied by Hammond \& Redekopp (1998) for a specific family of reverse flow profiles. They showed that for that particular family, absolute instability, which might lead to global dynamics if the bubble is large enough, will occur when the peak back flow velocity exceeds about $30 \%$ of the ambient free-stream velocity. In an appendix, they also looked at the effect of a free-slip boundary on the wake profile studied by Huerre \& Monkewitz (1985) and showed that a wall on the low-speed side of the layer generally leads to a requirement for more negative reverse flow velocities before the (inviscid) absolute instability sets in.

Table 1 provides a summary of these various (strictly parallel flow) cases studied previously using standard linear stability analysis. Actual wake flows are, of course, spatially developing and nonlinear effects are also usually important in development to any final periodic state. It is well-known that if there is a sufficiently large domain of absolute instability, a saturated global mode may appear. (This, for example, is the origin of the classical von Kármán vortex street.) The frequency (and nature) of such global modes can be explored by analysing the complex, nonlinear GinzburgLandau equation as a model of the flow (e.g. Pier et al. 1998; Pier \& Huerre 2001). Alternatively, it is possible to undertake (usually numerically) a linear stability analysis of full pre-computed solutions of spatially developing flows, to identify their stability and the frequency of any global mode which might result (e.g. Barkley \& Henderson 1996; Barkley, Gomes \& Henderson 2002). This has not yet been done for spatially developing versions of the wakes and mixing layers discussed earlier. Arguably the most straightforward way of answering the practical question of whether typical bubbles will, in fact, exhibit a global instability and if so, at what critical $R e$, is to conduct a physical experiment or to compute the full solution of the NS equations at various Reynolds numbers. Most of the experiments or computations on two-dimensional 
separated bubbles so far performed have been at Reynolds numbers sufficiently high for turbulence to be present also (e.g. Alam \& Sandham 2000; Manhart \& Friedrich 2001) and so are well beyond the $R e$ range within which the instability might first appear. There is certainly no doubt that many fully turbulent bubbles - e.g. behind a rearward facing step - contain a definite unsteadiness of a frequency much lower than that appropriate to the largest eddies which occur around reattachment (e.g. Cherry, Hillier \& Latour 1984; Kiya \& Sasaki 1985; Castro \& Haque 1987; Heenan \& Morrison 1998). There has been considerable debate about the precise nature of this unsteadiness and, as a result of multi-point pressure measurements and a review of the literature, Hudy, Naguib \& Humphries (2003) have recently suggested that an inherent absolute instability near the middle of the recirculation region provides the basic driver for the observed oscillations in bubble length.

The objectives in the work presented in this paper were essentially two-fold. First, we wished to pursue our earlier suspicion referred to above (in Castro 2002, hereinafter referred to as CA) that two-dimensional laminar separated wakes in a channel become susceptible to a global instability. In particular, using full NS computations, the aim was to find (for fixed channel width) the Reynolds number at which such a global mode first appears, the eventual saturated nature of that mode and whether it is similar to the long-time-scale unsteadiness identified in previous experiments at very much higher $R e$. Secondly, rather than engaging in an extensive and technically complex study of the spatially developing wake (using recently developed techniques such as those mentioned above) we wished to compare the computational results with the implications of straightforward linear stability theory for parallel flows, applied specifically to reversed flow profiles of the sort found in the numerical experiments. In making comparisons with the computations, we therefore expect to find no more than qualitative agreement at best. Only neutrally stable flows are considered. In addition, only two-dimensional flows are computed, which implies the assumption that the first instability to arise as $R e$ increases will be a two-dimensional one. This is usually, although not always, the case, but is definitely so for the cases listed in table 1 .

The numerical NS computations are initially presented and discussed in $\S 3$ and are then (in §5) considered in the light of the new results from the linear stability calculations. The latter were undertaken using, first, model shear-layer profiles of the form defined by (1.2), but for viscous cases and with symmetry conditions imposed on both sides of the layer, exactly as in the channel flow NS computations. The approach was similar to that used by Alam \& Sandham (2000) in that the investigation of the convective or absolute nature of the instability was done using Niew's (1993) method, which is a modification of Gaster's (1981) technique. Secondly, these calculations were supplemented by others which employed reverse flow velocity profile models closer to those actually found in the NS computations, just prior to the onset of the instability. A brief description of the method and presentation of the results of these linear stability calculations are contained in $\S 4$, and table 1 includes statements regarding this work, to show how it complements earlier related studies. Final discussion and conclusions are presented in $\S 5$. We start, in $\S 2$, with an outline of the numerical methods used for the NS computations.

\section{The numerical techniques}

The governing equations are the usual NS equations, given below and in which velocities are normalized by the upstream value, $U$, pressure is normalized by $\rho U^{2}$ and lengths are normalized by the (unit) half-width of the body generating the wake 


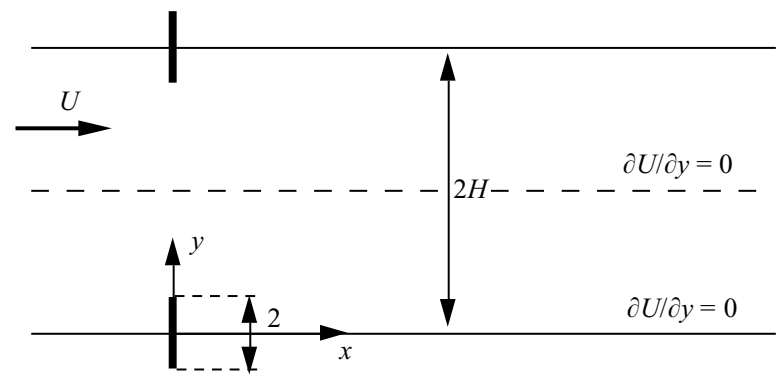

FIGURE 1 . Sketch of the geometry. Only the half-space $0 \leqslant y \leqslant H$ is computed.

(a simple flat plate throughout this work):

$$
\begin{gathered}
\partial_{t} \boldsymbol{u}+\boldsymbol{u} \cdot \nabla \boldsymbol{u}=-\nabla p+(1 / R e) \nabla^{2} \boldsymbol{u}, \\
\nabla \cdot \boldsymbol{u}=0 .
\end{gathered}
$$

Symmetry about $y=0$, the $x$-axis through the centre of the body, allows consideration to be restricted to the upper half of the flow field (i.e. $0 \leqslant y \leqslant H$ ), as illustrated in figure 1 . The boundary conditions are

$$
\begin{gathered}
x \rightarrow-\infty: \quad u(y) \rightarrow 1, \\
y=0, H: \quad \partial u / \partial y=0, \quad v=0,
\end{gathered}
$$

and, on the body surface,

$$
\boldsymbol{u}=0 .
$$

Equations (2.4) embody the imposed symmetry condition at $y=0$ and the zero-stress condition ensures that $y=H$ is a streamline. The downstream $x \rightarrow+\infty$ boundary condition imposed by the numerical scheme was normally one of zero gradient (on $u$ ). It was important to ensure that the upstream $\left(x_{u}\right)$ and downstream $\left(x_{d}\right)$ boundaries were sufficiently remote from the obstacle to have no effect on the solutions. With the length of the bubble defined by $L$, for steady cases, the previous work had confirmed that locating these at $x_{u}=-60$ and $x_{d} \geqslant 2 L$, respectively, was more than adequate. In the present unsteady cases, no special conditions were imposed at $x_{d}$, but numerous checks were done, by repeating computations with the boundary significantly further downstream. This was important since it has been shown that global modes can sometimes arise in numerical calculations because of wave reflections at the inlet or outlet boundaries (e.g. Lowery \& Reynolds 1986; Buell \& Huerre 1988). However, the checks demonstrated (for all results shown herein) that the quantitative characteristics of the global instability were not affected by boundary location. No doubt the computations could have been made somewhat more efficient by employing a more sophisticated outflow condition, allowing a reduction in $x_{d}$, but this was not deemed necessary.

Except for the time-dependence treatment, the numerical method used to solve these equations was identical to that described in CA for the earlier work, so need not be presented in detail. Suffice to say that (2.1)-(2.2) were discretized using a finite-volume approach on a staggered non-uniform grid and solved using a multi-grid version of a fairly standard iterative (ADI) method. For steady flow, integration of the momentum equations, (2.1), over a typical grid cell and collection of convective and diffusive contributions from the four cell faces leads as usual to

$$
a_{P} \phi_{P}=\sum a_{m} \phi_{m}+S_{P}^{\phi}
$$


where $\phi_{P}$ stands for the value of the $u$ or $v$ variable at the centre of the cell, $\phi_{m}$ the values at the centres of the four neighbouring cells, $S_{P}^{\phi}$ is a source term (containing the appropriate pressure gradient), the $a$ values are the multiplying coefficients containing the convective and diffusive flow rates, and the summation is taken over the neighbouring cells. The equations were solved iteratively in turn, along with a pressure perturbation equation derived using SIMPLER (Patankar 1980), which is more efficient that the well-known SIMPLE algorithm. As usual, under-relaxation was required to assure convergence; typical values of the under-relaxation factors were in the range 0.4-0.8 for the velocities and $0.2-0.4$ for the pressure correction.

For time-dependent computations, a second-order-accurate backward difference time-stepping scheme was used for the time-derivative term in (2.1):

$$
\frac{3 \phi_{P}^{n+1}-4 \phi_{P}^{n}+\phi_{P}^{n-1}}{2 \Delta t}
$$

with the effectively implicit equation for the flow variable $\phi_{P}$ at the centre of each cell at the new time level $(n+1)$ being given by a modified version of $(2.6)$, which can be written as

$$
\left(a_{P}+\frac{3 \Delta V}{2 \Delta t}\right) \phi_{P}^{n+1}=\sum a_{m} \phi_{m}^{n+1}+\left(S_{P}^{\phi}+\frac{4 \phi_{P}^{n}-\phi_{P}^{n-1}}{2 \Delta t}\right),
$$

where $\Delta V$ is the area of the control volume surrounding node $P$. At each time step, the identical iterative scheme was used to solve the equations, with similar convergence criteria being applied before moving to the next time step.

Central differencing was used for the diffusive terms and solution accuracy is then crucially dependent on the differencing of the convective terms. The monotonic scheme described by Leonard \& Mokhtari (1990) was used. This is an appropriate blend of central differencing and second-order upwind interpolation, with the scheme's stability maintained by including the second-order corrections in the source term. Further details can be found in CA, which also outlines the multigrid procedures used. As before, up to five hierarchical grid levels were used, with successively coarser grids being produced simply by removing every other node in both directions. Restriction to coarser grids used averages of near-grid neighbours, whereas prolongation to finer grids used bilinear interpolation. Generally, a W-cycle was used to visit the grids sequentially, with one or two post-smoothing iterations on each. Similar grid structures were used to those described in CA, with particular care being employed to maintain appropriate grid structure in the regions adjacent to the plate itself on each grid of the hierarchy. The grids were highly non-uniform to ensure adequate resolution around the plate, with typical mesh lengths there being $0.002(0.2 \%$ of the plate half-width), but with grid expansion factors kept below about 1.1 to minimize discretization errors arising from grid non-uniformity.

Solution accuracy for the steady-state case was addressed in CA where Richardson extrapolation was used and it was found, for example, that the fine-grid-solution values of $L$, the length of the separated region, and $C_{d}$, the plate drag coefficient, differed by less than $0.5 \%$ from those obtained by Natarajan et al. (1993) using an entirely different numerical method. Since very similar and in some cases identical grids were used for the present work, there is no reason to suppose that the unsteady solutions which demonstrate long-time-scale periodicity (which are those of particular interest in this paper) would be susceptible to (spatial resolution) errors any larger than these. However, assessment of the necessary time step required for accurate unsteady computations was obviously necessary. This will be addressed below as appropriate. 
Since the equations were solved in non-dimensional form, obtaining a solution at a new Reynolds number (on the same grid) was normally achieved simply by using a previous solution at another, not too remote, value of $R e$ as the initial condition and stepping immediately to the new value of $R e$. Not surprisingly, this usually led to initial transients, but their amplitude did not seem to depend strongly on the amplitude of the step change in $R e$. Using a more gradual ramp-like variation in $R e$ may arguably have led to a more rapid arrival at the stable state (whether steady or unsteady), but this was not tried; it would not, of course, have changed the nature of the final solution.

\section{The numerical results}

\subsection{Characteristics of the global-mode saturated state}

Presentation of the results of the time-dependent computations starts with an overview of the nature of the major (saturated) global instability found for a wide range of $H$ and $R e$. For sufficiently large values of these two parameters (precisely how large is explored later), a characteristic long-time-scale periodic behaviour was found, which continues unchanged for as long as the computation is run. The separated region gradually elongates and then begins to 'pinch' near its downstream end, until eventually a secondary 'bubble' is released and moves off downstream. This release is naturally accompanied by a reduction in the downstream distance to the first stagnation point (i.e. the length of the main bubble reduces suddenly) and the whole cycle begins again. The behaviour is illustrated in figures $2-4$, for the specific (but very typical) case of $H=10, R e=200$. Note the difference in scale of the axes - the eddy is much more elongated than it seems on these figures. An initial glance at figure 3 will indicate that the main bubble gradually lengthens (figures $3 a-c$ ) and then more suddenly contracts (figure $3 d$ ), as a secondary bubble is 'shed'. In figure 2, the distance to the most downwind stagnation point on $y=0, L^{\prime}$, is shown as a function of time, with figure 2(a) showing the results of a computation which started from a solution previously obtained using the steady-state version of the code. (Such solutions could not always be obtained, of course, but judicious choice of relaxation and multi-grid parameters did in this case produce one.) It is very clear that the steady solution is, in fact, unstable and eventually the characteristic unsteady behaviour develops. These results were obtained using the finest grid $(754 \times 210)$ and with a time step of $\Delta t=5$.

In figure $2(b)$ the result of a similar calculation using the next coarsest grid $(378 \times 106)$ is shown, but this one was started from an $R e=150$ solution obtained using the time-dependent code (but found to remain steady). The period of the regular oscillations in $L^{\prime}$ is a little less than was found with the finer grid, but the difference is below $4 \%$. To check the influence of the time step, a further run was undertaken, starting with the coarser-grid solution at $t=14800$ and running to about $t=16000$ but using $\Delta t=0.5$ (i.e. a factor of ten lower) and the result for this is included in figure $2(b)$. It can be seen that this makes very little difference to the period, but tracks the downstream secondary bubble a little further. Figure 2(c) expands a section of the $\Delta t=0.5$ solution and highlights the motion of the main bubble (in bold) and that of the secondary bubble (the forward and upward pointing 'horns', whose lower and upper boundaries mark the locations of the upstream and downstream stagnation points, respectively, of this secondary bubble). The single large bubble slowly grows and then splits (at $t=15111,15518$ and 15925 in figure $2 c$ ), with the much shorter secondary bubble moving off downstream, reducing in size and eventually disappearing altogether (at around $t=15251,15658$ and 16065). Notice that for this case, the 

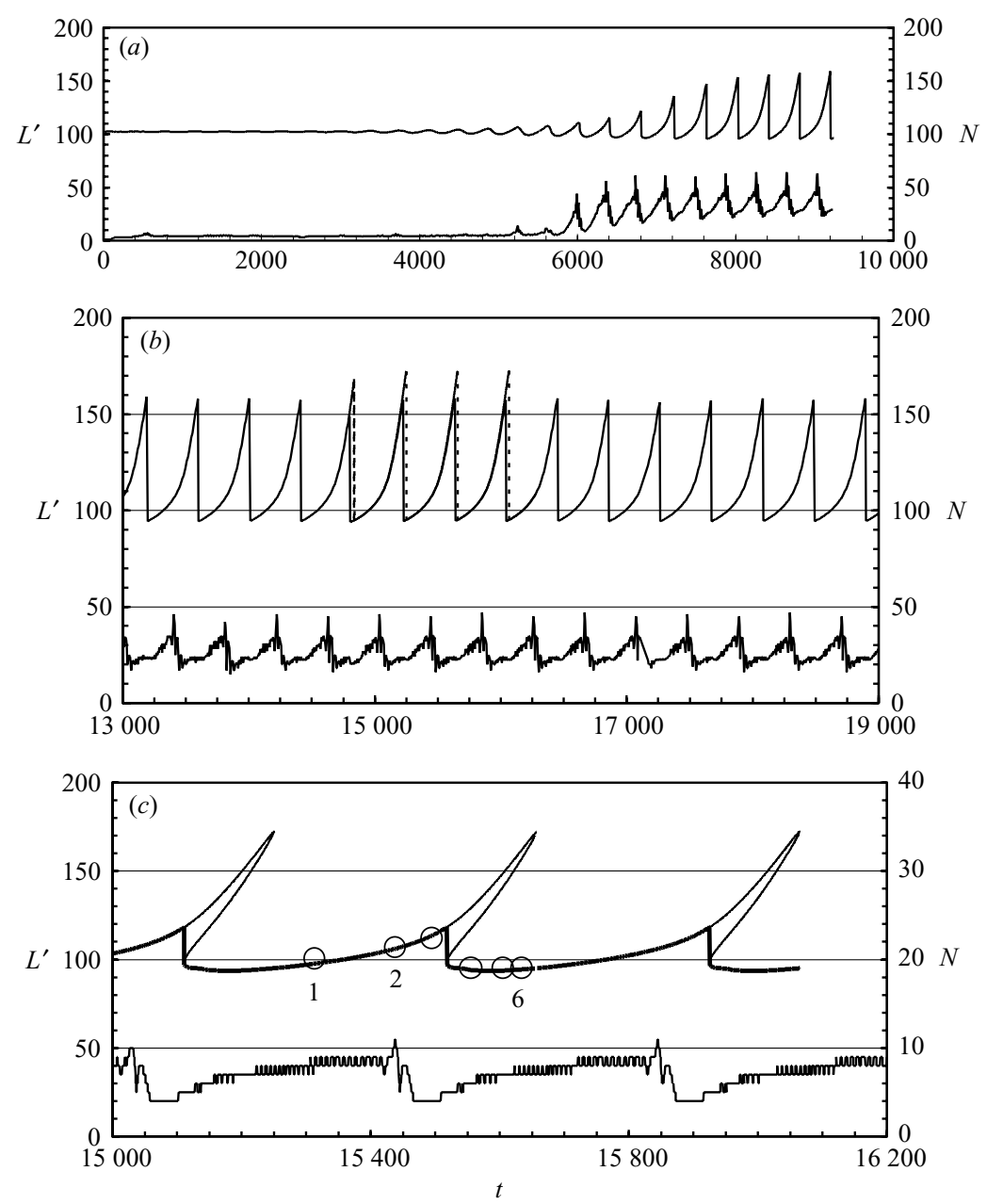

FIgURE 2. $H=10, R e=200$. Behaviour of the stagnation point locations. (a) Started from steady solution on grid $1, \Delta t=5$. (b) Grid 2, $\Delta t=5$ (solid line), $\Delta t=0.5$ (dotted line). (c) Grid 2, $\Delta t=0.5$, expanded portion. Bold solid line shows main bubble behaviour. Circles identify times for the contour plots of figures 3 and 4 . Lower lines in each graph refer to number of cycles for convergence at each time step (right-hand axes refer).

period of the underlying oscillation is very long, at around 407 time units. We discuss later what sets this period.

In each of the three plots in figure 2, the lower data refer to the number $(N)$ of iterative multi-grid cycles required to achieve convergence at each time step. With $\Delta t=5$, nearly 50 cycles are eventually needed at the 'worst' part of the period - a little prior to the appearance of the secondary downstream bubble, figure $2(b)-$ and rather more in the case of the finer grid (figure $2 a$ ). Reducing the time step to $\Delta t=0.5$ naturally leads to far fewer cycles being required, as shown in figure $2(c)$, where over practically all the oscillation period, fewer than 10 cycles are required. Of course, $N$ depends on the convergence criteria employed and care was needed to ensure that the value of the equation residuals deemed acceptable for a converged solution was sufficiently low. It turned out that there was sometimes considerable leeway here; indeed, even enforcing a maximum $N_{m}$ value, so that the calculation 
(a) 1
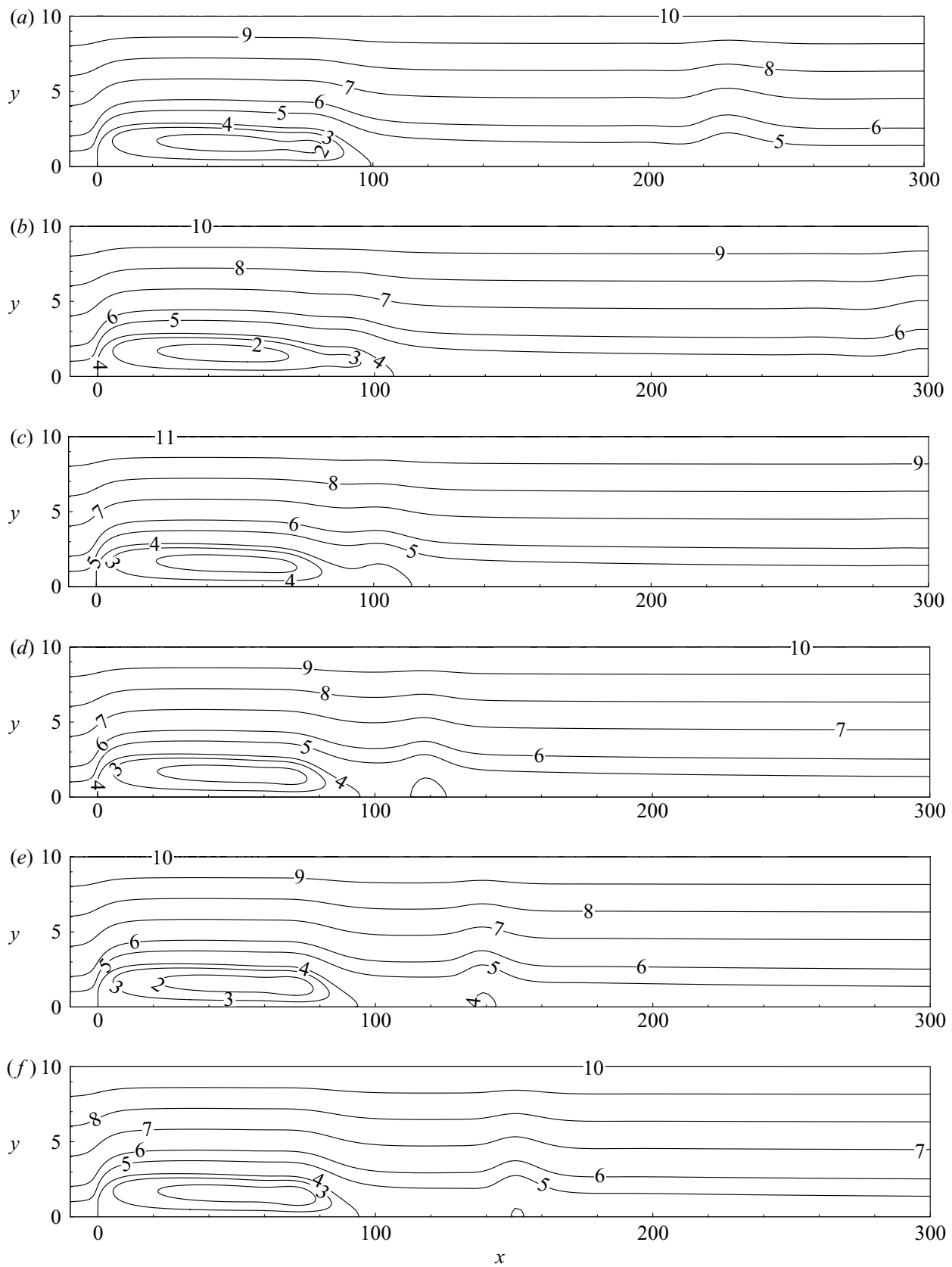

FiguRE 3. Streamlines for $H=10, R e=200 .(a)-(f)$ correspond to times labelled 1-6 on figure 2(c). Contour values (labels): $-0.6(1),-0.4(2),-0.2(3), 0(4), 1$ (5), 2 (6), 4 (7), 6 (8), 8 (9), $10(10)$.

automatically proceeded to the next time step after $N_{m}$ multi-grid cycles whatever the values of the current residuals, often led to no discernible difference in the overall behaviour of the flow. On the other hand, there were many cases where double precision calculations along with demanding convergence criteria were necessary to avoid apparent instabilities which could otherwise appear. These were simply the 

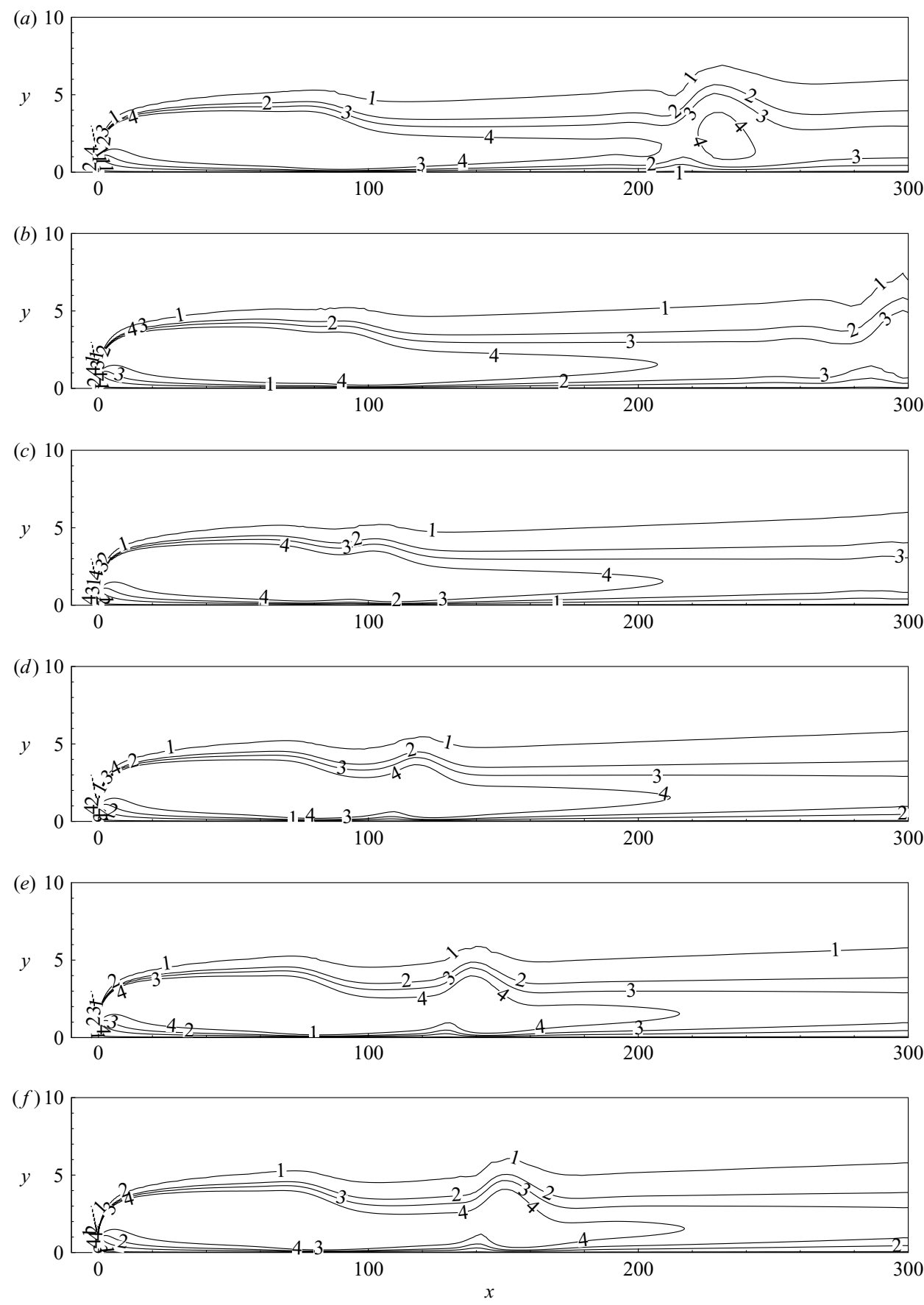

FIGURE 4. Vorticity contours corresponding to the streamlines in figure 3 , for $H=10$, $R e=200$. Contour values (labels): 0.005 (1), 0.05 (2), 0.1 (3), 0.2 (4).

result of inadequate convergence and could not be reduced if running under single precision, however strict the convergence requirement. In these more taxing cases, the sum of the absolute values of all the residuals was typically required to be below $10^{-12}$, for each equation solved (i.e. both momentum equations and the pressure correction 
equation). Such levels correspond to around the machine (double precision) rounding errors at each individual computational cell.

Figures 3 and 4 show streamlines and vorticity contours at selected times (indicated on figure $2 c$ ) during a single cycle. These clearly illustrate the nature of the 'pinching' process at the rear end of the main bubble, the subsequent movement downstream of the resulting secondary bubble, and its eventual disappearance. Even after disappearance of the bubble (e.g. figures $3 a, 4 a$ ) the characteristic vorticity 'heap', initiated during the pinching process, remains, and continues to be swept downstream whilst slowly diffusing. Note particularly that, as far as the main bubble is concerned, the major activity occurs downstream of its first half; this is most clearly seen in figure 4, which indicates that movement of vorticity contours is largely absent upstream of $x=50$. In fact, remarkably, the obstacle's drag coefficient, $C_{d}$, remains fixed (to four significant figures at least) throughout the entire oscillation period, indicating that the downstream unsteady processes have no influence on the separation at the plate tip and, in particular, on the amount of vorticity being shed into the wake. It should be emphasized that moving the downstream boundary further downstream (it was at $x=300$ for the computations discussed thus far) had no influence on the behaviour of the bubble, so it is certain that this global mode is not a result of the downstream boundary condition nor its location. Naturally, this boundary condition does influence the details of the flow near it (recall that a zero axial gradient condition was imposed here), although this is hardly noticeable in figure 4.

\subsection{Behaviour near the critical Reynolds number}

It is natural to ask at this stage how the flow changes as the $R e$ increases through the value at which the global instability discussed above first appears and, indeed, what the value of $R e_{c g}$ actually is. We use this notation $\left(R e_{c g}\right)$ for the critical Reynolds number, anticipating the discussion in $\S 4$, since the global mode will not occur until $R e$ significantly exceeds the value required for the first appearance of absolute instability, which we term $R e_{c a}$. This will in turn be greater than the critical $R e$ required for first appearance of a convective instability, which we term $R e_{c c}$. By gradually increasing the Reynolds number, starting from a value which gave a steady flow, until oscillations in $L$ and in the velocity at a selected location in the flow field first began to appear, $R e_{c g}$ was determined for $H$ in the range $3 \leqslant H \leqslant 20$. The results are illustrated by again considering the $H=10$ case. Time-dependent runs for $R e \leqslant 180$ always gave eventually steady solutions. Figure $5(a)$, for $R e=170$, illustrates the typical behaviour; in this case the run started from a steady $R e=150$ solution and, after some initial oscillations, $L$ clearly settles down to a steady value, identical to the one which could be obtained separately by using the steady-state version of the code. (Note that once the secondary bubbles are no longer shed, after about $t=11000$ in figure $5 a, L^{\prime}=L$ ). Moving then to $R e=180$, however, gave a rather different behaviour, as shown in figure $5(b-d)$. The initial oscillations in this case, although appearing to die away, attenuated extremely slowly; figure $5(c)$ shows the peak-to-peak amplitude $(\Delta L)$ of the oscillations in $L$ and the results suggest an eventual amplitude of about $0.8-$ i.e. some $0.9 \%$. The result of an alternative computation which started from the converged solution obtained using the steady version of the code is shown in figure $5(d)$, where it is clear that oscillations in $L$ eventually build up and, indeed, have a steady amplitude close to that anticipated from the exponentially decaying fit to the data in figure $5(c)$.

A supercritical bifurcation would be expected to yield a quadratic behaviour in the saturated amplitude of the oscillations of a flow variable at Reynolds numbers 

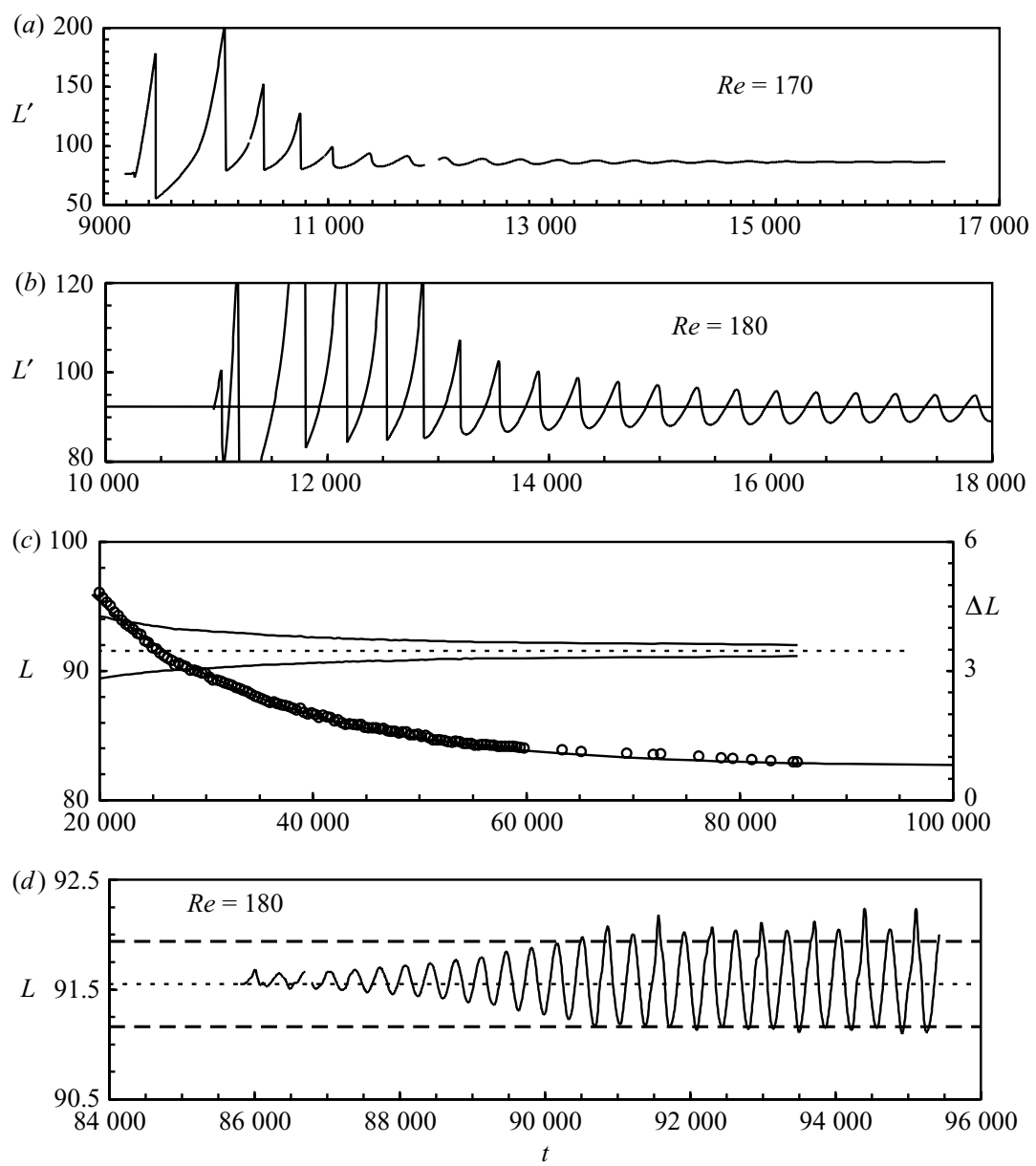

Figure 5. $H=10$, grid 1 . (a) $R e=170$, starting from a steady $R e=150$ solution. (b) $R e=180$, started from the steady $R e=170$ solution; the horizontal line denotes the steady solution $L$. (c) Variation of the amplitude of oscillations in $L$ at much later times; dashed line is the steady solution and the two solid lines show the maximum and minimum $L$ in each cycle; the circles (right-hand axis refers) show the peak-to-peak amplitude. $(d) R e=180$, starting from its steady solution, the short-dashed line; long-dashed lines refer to the eventual peak-to-peak levels deduced from the (shown) exponential fit to the circles in $(c)$.

just above the critical. Figure 6 shows the behaviour of $\Delta L^{2}$ from computations for $R e \leqslant 190(\Delta L$ is the normalized peak-to-peak variation in bubble length). In addition, the corresponding values of $\Delta u^{2}$ are shown, where $\Delta u$ is the peak-to-peak oscillation amplitude of the axial velocity at the arbitrarily chosen $(x, y)$ location of $(100,2)$, i.e. just downwind of the bubble's rear stagnation point. The data suggest that the instability is indeed of supercritical type. A detailed study of the flow near the critical condition - using an appropriate Landau model, for example - would be instructive, but is beyond the scope (and the emphasis) of the present work.

One might expect oscillation amplitudes (e.g. $\Delta L$ or $\Delta u$ ) at a fixed $R e$ to depend on the domain width, $H$. Figure 7 shows how $H$ affects the variation in length of the main bubble over a complete oscillation cycle, for $R e=200$. The data demonstrate that as $H$ increases, the amplitude of the oscillation increases rapidly, suggesting that 


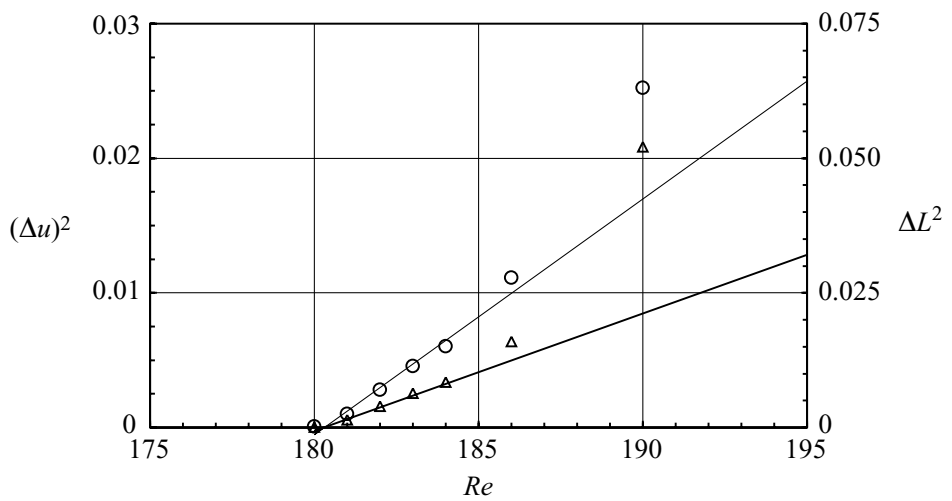

FiguRE 6. Behaviour of amplitude oscillations in bubble length (triangles, right-hand axis, normalized by $\left.L^{2}\right)$ and velocity at $(100,2)$ (circles, left-hand axis). Straight lines drawn through the data for $R e<185$.

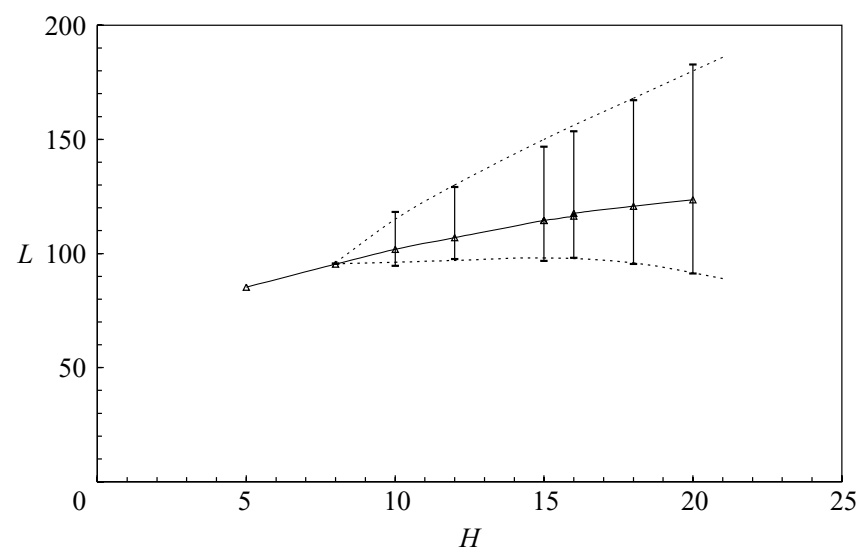

FIGURE 7. Peak-to-peak values of $L$ at $R e=200$ (dotted lines through data), compared with steady values (triangles, line added for clarity).
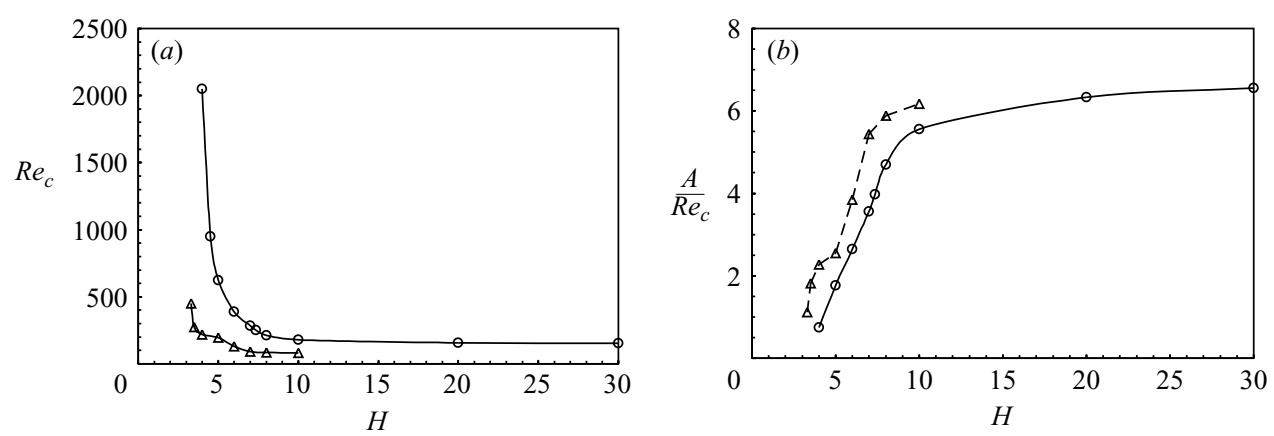

FiguRE 8. (a) Critical Reynolds number for global instability from the NS computations $\left(R e_{c g}\right.$, circles) and for absolute instability in the OS calculations $\left(R e_{c a}\right.$, triangles). (b) Inverse of $\operatorname{Re}_{c}(A / \operatorname{Re}$ with $A=1000$ for NS or 500 for OS, chosen for convenience).

the critical $R e$ falls as $H$ increases. Figure 8(a), which shows how $R e_{c g}$ varies with $H$, confirms this. Consider for the moment the data identified by circles (the other data refer to the Orr-Sommerfeld linear stability calculations and will be discussed 


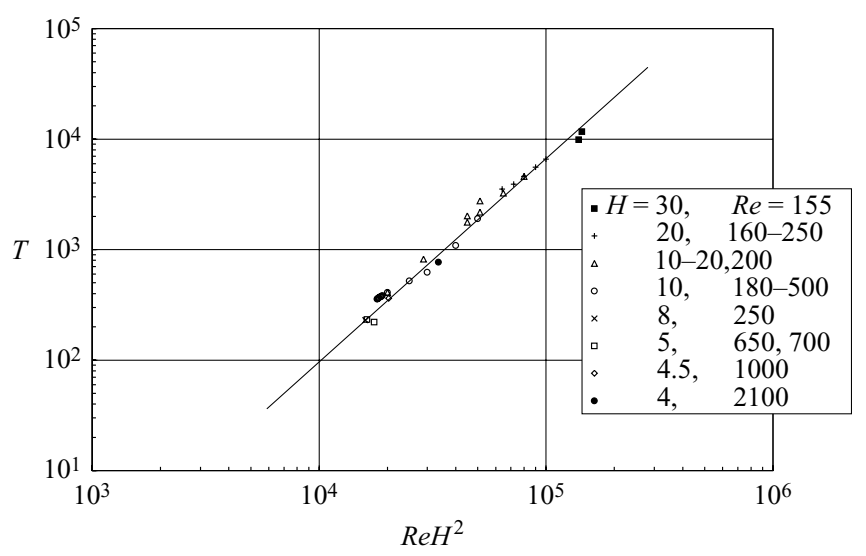

Figure 9. Period of the global oscillation, as a function of $\mathrm{ReH}^{2}$.

later). As $H$ falls, $R e_{c g}$ rises, eventually quite rapidly as $H$ approaches 3 . Indeed, the computations for $H=4$ did not yield an unsteady solution even at $R e=2000$, by which time the bubble length had grown to $L=840$; computations at significantly higher $R e$ for that $H$ were not attempted. Figure $8(b)$ shows the inverse of $R e_{c g}$ and helps to clarify that there is probably a lower limit on $H$, quite close to $H=3$, below which instability never occurs. This is consistent with the results of the linear stability calculations discussed in $\S 4$ (and also shown in figure $8 b$ ). Note that no attempt was made to determine $R e_{c g}$ with great precision. Typically, the change in $R e$ used to move from a steady to an unsteady solution was some $1-2 \%$. For the higher of the two $R e$, oscillations in $L$ would then have amplitudes of at least $10 \%$ whereas, at the lower $R e, L$ would be constant to within machine rounding error. Only for the $H=10$ case discussed above was $R e_{c g}$ resolved to greater accuracy (better than $0.5 \%$, as suggested by the data in figure 6) and, in this case, doubling the spatial resolution and/or reducing the time step by two orders of magnitude did not change the results qualitatively.

It was noted above that the time scale of the characteristic global mode is large (407 time units for $H=10, R e=200$ ). Now the total vorticity $\Omega$ inside the eddy (of area $A$ ) is given by $\Omega \sim \omega_{e d} A \sim \omega_{e d} H R e$, where $\omega_{e d}$ is a characteristic vorticity within the eddy. For an eddy of length $L$, the rate $R_{d}$ at which this vorticity diffuses out of the bubble - across the shear layer bounding the bubble - will be given by $R_{d} \sim(1 / R e)\left(\omega_{e d} / H\right) L \sim \omega_{e d} / H$, assuming that the eddy width scales with $H$ and $L \sim R e$. Thus, the time period $T_{p}$ for significant removal of vorticity from the eddy, which might be expected to control the time scale of the bubble oscillations, will be given by $\Omega / R_{d} \sim R e H^{2}$. Figure 9 shows a variety of data for $4 \leqslant H \leqslant 30$ and $155 \leqslant R e \leqslant 2100$ which certainly seems to confirm that $T_{p}$ is functionally dependent on $\mathrm{ReH}^{2}$, although the log-log plot used suggests a slope closer to 2 than to 1 . We return to this issue in $\S 5$.

As $R e$ is increased beyond the critical region (with $H$ fixed), both the amplitude and, as implied by the above argument and figure 9, the period of the oscillations increase. The secondary eddy becomes larger as it is released from the main eddy and it survives for longer distances downstream. With further increases in $R e$, we would expect further instabilities, perhaps of the usual Kelvin-Helmholt $(\mathrm{K}-\mathrm{H})$ kind, leading eventually to transition. For the $H=10$ case, there was some evidence that this began to occur between $R e=250$ and 300 - higher-frequency lower-amplitude 
oscillations, appeared on top of the periodic fluctuations in flow variables caused by the initial global mode. Not surprisingly, we found that using large enough time steps tended to smooth these oscillations, but the results are not presented here since at this $R e$ they may in any case be non-physical (the code is solving the two-dimensional equations); they sufficed, however, to illustrate the point that at this $H$ the genuinely two-dimensional global mode sets in at a lower $R e$ than needed to trigger the usual transition process. No attempt was made to identify precisely the critical Reynolds number for the appearance of the higher-frequency modes, but it may be noted that, like $R e_{c a}$, we found that it rises with decreasing $H$. At $H=4$, for example, although no global mode of the kind seen at higher $H$ set in until around $R e=2100$, use of a much smaller time step $(\Delta t=0.05)$ did lead to instability and this, in fact, occurred at much lower $R e$ : for $R e>495$, clear instabilities were apparent in the shear layer upstream of the end of the bubble. Likewise, at $H=3$, the same small time step led to shear-layer instabilities for $R e \gtrsim 550$. These instabilities are likely to be associated with the regular $\mathrm{K}-\mathrm{H}$ modes and were not studied further; the crucial point in the present context is that for low enough $H$ (roughly, $H \lesssim 6$ ), as $R e$ rises, they appear before the low-frequency global mode, whereas at high enough $H$, the latter appears first.

\section{Stability calculations and comparisons}

We turn now to presentation of the linear stability calculations, noting the importance of distinguishing between convective and absolute instability (Gaster 1963). This was done using Niew's (1993) method, which is a modification of Gaster's (1981) original technique in which the impulse response of a particular velocity profile is found from a complex summation of the temporal dispersion relation given by

$$
G(x, t)=\sum_{j=1}^{J} \mathrm{e}^{\mathrm{i}\left(\alpha_{j} x-\omega_{j} t\right)} .
$$

This expression is a discretized version of the full integral relation, simplified by recognizing that the integral will be dominated by the exponentially large elements so that the multiplying amplitudes describing the structure of the eigensolutions will only provide weak modifications; they are therefore all taken as unity. The complex frequency $\omega$ for each real value of the wavenumber (discretized by) $\alpha_{j}$ is computed from the Orr-Sommerfeld equation for the perturbation streamfunction $\phi$, derived by assuming locally parallel flow and expressed as

$$
\left(U-\frac{\omega}{\alpha}\right)\left(\phi^{\prime \prime}-\alpha^{2} \phi^{\prime \prime}\right)-U^{\prime \prime} \phi=\frac{\mathrm{i}}{\operatorname{Re} \alpha}\left(\alpha^{4} \phi-2 \alpha^{2} \phi^{\prime \prime}+\phi^{\prime \prime \prime \prime}\right),
$$

where $U$ is the mean velocity profile and derivatives with respect to $y$ are shown by primes. The summation in (4.1) is carried out over the unstable range of waves (i.e. over the range, $j=1 \rightarrow J$, of real $\alpha_{j}$ which yields positive values of imaginary $\left.\omega_{j}\right)$. For a given velocity profile, $R e$ was gradually increased to find the first value at which such a real $\alpha_{j}$ just appeared. This is the critical $R e$ at which the flow first becomes unstable, but if the subsequent calculations of real $G(x, t)$ at $x=0, G_{r}$ say, show oscillations which decay with time, this instability is convective and the critical $R e$ is denoted by $R e_{c c}$, as defined earlier. Further increases in $R e$ will lead to a point when $G_{r}$ oscillations begin to increase in time and the $R e$ at which this first occurs is denoted by $R e_{c a}$, the critical $R e$ for absolute instability. We emphasize that 


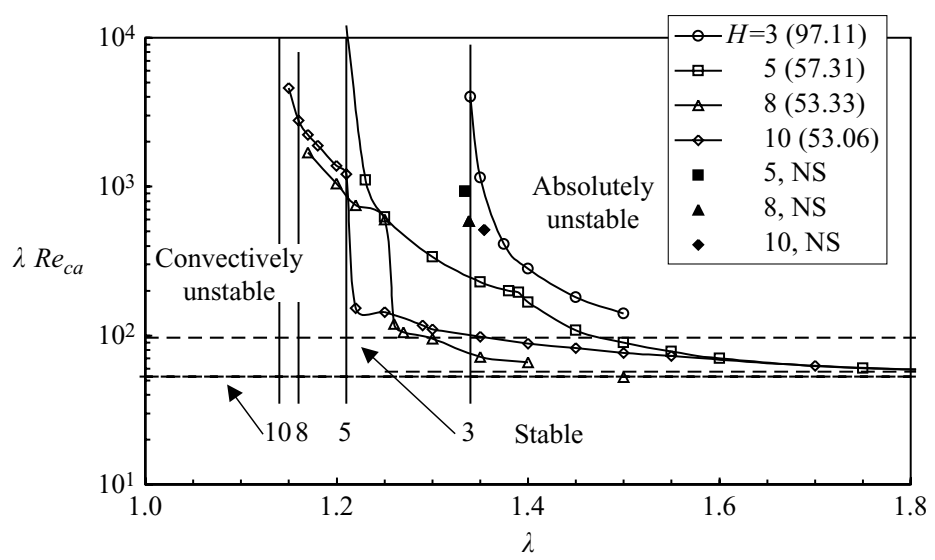

FIGURE 10. Variation of critical Reynolds number for absolute instability. Horizontal (chain) lines refer to Reynolds number for convective instability $\left(R e_{c c}\right)$, with relevant $H$ values indicated in the figure and $R e_{c c}$ values (in brackets) in the key. Vertical (solid) lines denote limits in $\lambda$ below which absolute instability never occurs, with relevant $H$ values shown below them. Solid symbols refer to critical $R e$ for global instability, from the NS computations (i.e. $R e_{c g}$ ). Note that the $R e$ values plotted here are based on the maximum velocity difference, i.e. the ordinate is $\lambda R e_{c a}$.

the technique identifies only the onset of the most rapidly growing mode and is, of course, limited to the assumption of parallel flow. We do not, therefore, anticipate a quantitatively close comparison between the results and those from computations of the full NS equations. The only justification for such a comparison is that linear theory has been shown in the past to give useful indications of the initial processes leading to global instability (despite the probable importance of nonlinear effects) and we proceed in the hope that the qualitative behaviour may indeed be similar, as, in fact, turns out to be the case.

To allow direct comparisons with their results, this procedure was used first for the model velocity profile studied by Hultgren \& Aggarwal (1987) (hereinafter referred to as HA) and defined by (1.2), for which they found $R e_{c c}=53$. It should be emphasized that this result refers to the anti-symmetric streamfunction modes, or what could be called 'in-phase vortex shedding', rather than the symmetric modes which correspond to the more usual out-of-phase (von Kármán) shedding. HA concentrated their study on this latter case, for which the critical Reynolds number is much smaller. In the present work, this mode is suppressed by the imposition of the free-slip symmetry plane at $y=0$. As noted earlier, HA's $R e$ was based on the maximum velocity difference across the shear layer, $\lambda U$, and was independent of $\lambda ; \lambda-1$ is the peak negative velocity on the low-speed side of the layer. In the present calculations, unlike those of HA, a free-slip upper boundary at $y=H$ was also imposed and the location of this boundary provides a second parameter, just as in the NS computations described earlier. It was found that for $H=100,10,8,5,4,3.5$ and $3, R e_{c c}=52.99,53.06,53.33$, 57.30, 64.68, 74.15 and 97.11, respectively. The excellent agreement between the result for the largest $R e$ value and HA's result (53) provides confidence in the codes used for the computations, which were appropriately modified versions of those used by Alam \& Sandham (2000). Note how reducing $H$ leads to significant increases in the critical Reynolds number for convective instability.

In contrast to $R e_{c c}$, it was found that $R e_{c a}$ is dependent on $\lambda$. Figure 10 shows the behaviour of $R e_{c a}$ for $3 \leqslant H \leqslant 10$. Note that for each $H$, there is a lower limit on $\lambda$, 
$\lambda_{c}$ say, below which although the flow may be convectively unstable (for $R e>R e_{c c}$ ) it never becomes absolutely unstable. These limits are indicated on the figure and it is clear that $\lambda_{c}$ falls as $H$ increases. In other words, as the flow becomes more constricted by the upper boundary, not only does $R e_{c c}$ increase, but also higher values of reverse flow velocity are required before the flow can ever become absolutely unstable. Recall that the Tanh profile (1.1) was shown by Huerre \& Monkewitz (1985) to be inviscidly absolutely unstable for $\lambda>1.136$. Despite the slightly different form of the mean velocity profile the present (viscous) calculations are consistent with this - figure 10 shows that for $H>10, \lambda_{c}$ must be below about 1.14.

Figure 10 also shows that for a fixed $\lambda$, decreasing $H$ leads to increasing values of $R e_{c a}$. This too is consistent with the NS computations; the $R e_{c g}$ results in figure 8 were discussed earlier and have exactly that trend. The NS computations showed that the effective $\lambda$ values were almost always in the region $1.3-1.35$, in a wide $R e$ range bracketing $R e_{c a}$ and independent of $H$. For example, at $H=3$ and $R e=200$, the velocity at the axial location around the centre of the eddy varied from 2.162 at the upper boundary to -0.684 at the lower boundary. This corresponds to $\lambda=1.316$ $(1+(0.684 / 2.162))$ and the equivalent $R e$ (scaled as for figure 10) is about 570 . Note that the re-scaling also has to include adjustment for the actual wake half-width, which for this particular case required another multiplying factor of about 1.5. For $H=5$, the effective $\lambda$ around the middle of the eddy, at the Re just below that at which the instability first appears, was found to be about 1.335 and figure 10 implies that for such a case $R e_{c a}$ is about 280 . In fact, the NS computations remained globally stable until about $R e=600$ which, after the appropriate scaling, is equivalent to 1115 and is thus about four times what might be expected from the stability analysis. Similar differences were apparent for the higher $H$ cases and are not unexpected - it has been noted before that we only expect a global mode to appear in a real case if there is a sufficiently long axial region over which conditions for local absolute instability are satisfied (e.g. Hammond \& Redekopp 1998; Huerre \& Monkewitz 1990). In our NS computations, we cannot expect to identify the intermediate transition to local absolute instability; recall that $R e_{c g}$ denotes the point at which a self-sustained global mode first appears and that will occur only after a sufficiently large part of the flow has become absolutely unstable.

A further feature of figure 10 is the relatively sudden jumps in $R e_{c a}$ for $H=8,10$ around $\lambda=1.2-1.25$, which follows a trend initially discernible in the $H=5$ results where there is a clear kink in slope around $\lambda=1.4$. These jumps occur around the $\lambda$ values for which the period, $T_{c a}$, of the dominant mode at the critical Reynolds number, $R e_{c a}$, reaches large values. The periods deduced from the $G_{r}$ data have maximum values which are at least an order of magnitude greater than values for $\lambda$ remote from the peak positions. In fact, this made determination of the critical Reynolds number difficult; very low frequency modes require $G_{r}$ summations (from (3.1)) over long times - so long that serious precision issues arise because of the size of the argument in the exponential. The accuracy of critical Reynolds numbers above a few hundred is therefore questionable. Figure 11 shows the inverse periods at $R e_{c a}$ for each $H$. For $\lambda$ values lower than those which gave the maximum periods, the sign of the periods has been changed and the figure suggests that rather than having a simple peak in $T_{c a}$ the periods asymptote to infinity at a specific $\lambda$ (about 1.64 in the case of $H=3$, for example). This might imply that solution branches either side of this asymptote are in some way fundamentally different, particularly in the case of the higher $H$ values where there are sudden jumps in $R e_{c a}$ (figure 10), but a full explanation would require a much more detailed study. 


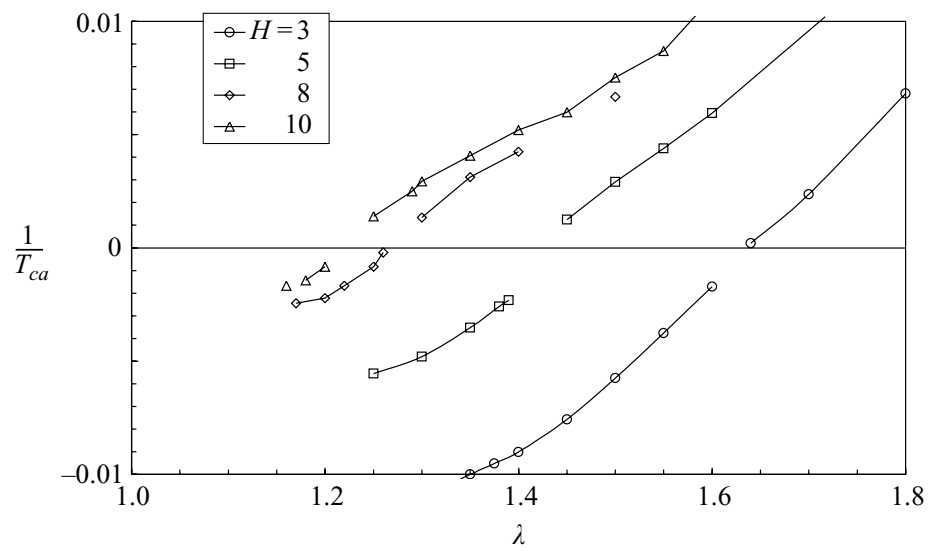

FiguRE 11. Inverse period, $1 / T_{c a}$, of the dominant mode at the critical absolute Reynolds number, $R e_{c a}$. Note the change of sign for $\lambda$ values below the values which give peak $T_{c a}$.
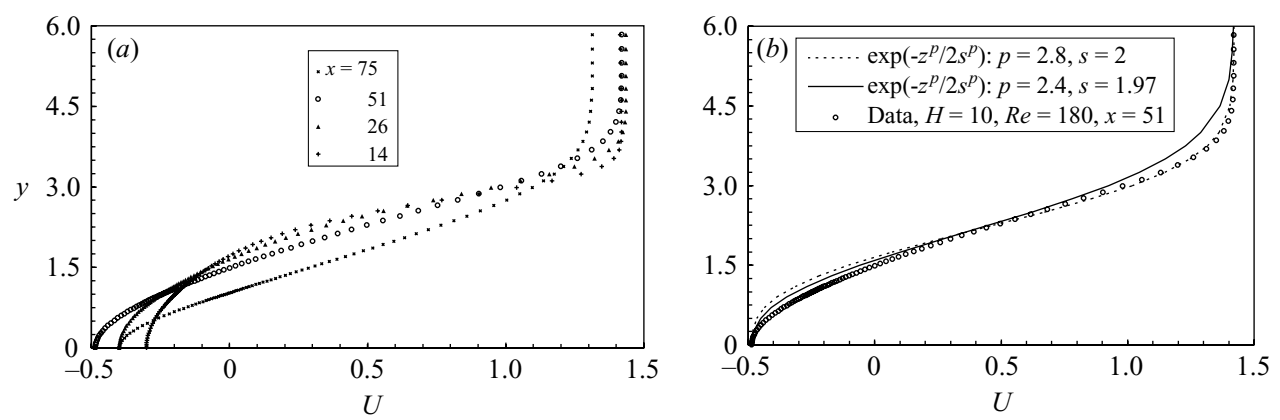

Figure 12. $U$-profiles from the steady (unstable) solution for $H e=10, R e=180$. (a) From the NS computations; $(b) \mathrm{e}^{-y^{p} / 2 \sigma^{p}}$ fits for the $x=51$ profile, for two values of $p$.

Since the form of the typical velocity profiles within the separated bubble computed by the NS code could not generally be modelled particularly accurately by the profile used for the stability calculations (equation (1.2)), some supplementary calculations were undertaken for profile shapes more closely matching the former. Results will not be presented in detail, but some comments are appropriate. We consider $H=10$, $R e=180$ as an example of a case just beyond the global stability limit as suggested by the NS computations. Figure 12(a) shows typical velocity profiles in the bubble, obtained from the steady (although just unstable) solution. Note that in this case, $L=92$. It was found that velocity profiles in the region $x<L$ could be reasonably well fitted using $\exp \left(-y^{p} / 2 \sigma^{p}\right)$ shapes, with $p$ in the range $2<p<3$ and depending on $x$, rather than fixed at $p=2$ as in (1.2). Figure $12(b)$ shows fits to the profiles at $x=51$, obtained either by ensuring that the peak velocity gradient matched that of the computed profile (which gave $p=2.4$ and $\sigma=1.97$ ) or, more freely, by adjusting $p$ and $\sigma$ to produce the best visually satisfactory fit (giving $p=2.8, \sigma=2$ ). The latter gives a noticeably better fit in the outer region of the profile, whereas the former does better in the inner region. Using such profiles in the stability calculations showed that in the upstream portion of the bubble, the profile was convectively, but not absolutely unstable even at very large $R e$. The profile at $x / L=0.5$, however, became absolutely unstable around $R e=50$. This result is much lower than the $R e_{c g}=180$ found by 
the NS computations, but this is entirely consistent with the well-known fact, noted earlier, that a significant portion of the flow must be beyond the absolutely unstable limit before a global mode can be expected to develop. (Of course, NS results for $R e=50$ gave profiles with much weaker backflow in the bubble and would thus be stable.) A further test of the general relevance of the results obtained with the HA model profile, defined by (1.2), was done by repeating the earlier calculations (whose results are shown in figures 10 and 11) for the same values of the backflow parameter, $\lambda$, but with the exponent, $p$, set at 2.8 (a typical 'worst case' of departure from a Gaussian profile) and with $\sigma$ then chosen to give the same total mass flux. The shapes of the $R e_{c a}-\lambda$ curves were essentially the same as those shown in figure 10, with lower limits to $\lambda$ below which the flow never became absolutely unstable very close to those shown, although the critical Reynolds numbers were significantly lower at all $\lambda$. We conclude that while the precise shape of the velocity profile does affect the critical Reynolds numbers, it does not change the way in which $H$ alters the occurrence of absolute instability.

\section{Concluding discussion}

It was noted in $\S 1$ that it has long been known that fully turbulent separation bubbles - those behind backward facing steps or near the front of blunt rectangular plates for example - often contain very low frequency fluctuations. These are often associated with shear layer 'flapping' (e.g. by Cherry et al. 1984; Kiya \& Sasaki 1985), with an associated quasi-periodic extension and collapse of the bubble, and typically have time scales some 5-10 times greater than those associated with the passage of large vortex structures within the shear layer around reattachment. The latter always occurs in a fully turbulent bubble, of course, but the presence of the former seems to depend on the nature of the bubble. In the work cited above, maximum backflow velocities were quite large $\left(U<-0.2 U_{\infty}\right)$ and this is more typical of long bubbles. However, if the backflow velocity is small compared to the free-stream velocity, this low-frequency component seems generally to be absent. This is evident from a number of experimental studies and also in more recent DNS computations of separated bubbles on a flat wall, arising from an adverse pressure gradient. Alam \& Sandham's (2000) bubble, for example, was relatively short and had maximum negative velocities above $15 \%$ of the free-stream velocity for less than $1 \%$ of the time. Whilst they noted large-scale structures being shed from the bubble, there was no hint of a very low-frequency (global) mode, which was consistent with their conclusion from linear stability analyses of the relevant velocity profiles: the bubble could be classified as convectively unstable because (steady) reverse flow velocities below $-0.15 U_{\infty}$ were required for absolute instability. Similarly, $\mathrm{Na} \&$ Moin (1998) found in their DNS of a (short) separation bubble that $U>-0.06 U_{\infty}$ everywhere and they did not identify a low-frequency global mode.

Pauley, Moin \& Reynolds (1990), in their experimental study of a laminar boundarylayer separating under a strong adverse pressure gradient, found a quasi-periodic shedding whose frequency, when expressed as

$$
f^{*}=\frac{1}{2} \pi \frac{\delta_{w} f}{U_{a v}},
$$

where $\delta_{w}$ and $U_{a v}$ are the vorticity thickness and the average velocity in the shear layer, respectively, was about 0.21 - very close to the most amplified frequency in an inviscid (parallel flow, linear) analysis of the Tanh profile (Michalke 1965). However, this 
frequency is about the same as the frequency found by Kiya \& Sasaki (1985), Cherry et al. (1984), Castro \& Haque (1987) and others, and associated with the large-scale shedding around reattachment. It is substantially higher, by a factor of some $5-10$, than the very low-frequency content found by all these authors at locations in the upstream half of their bubbles. This is consistent with the conclusions of Hudy et al. (2003), who identified a source near the centre of their bubble, producing upstream and downstream travelling disturbances at the much lower frequency of about $f^{*}=0.045$. Partly on the evidence of additional, more recent experimental data (Heenan \& Morrison 1998) they argued that this was evidence for 'an absolute instability zone, or self-sustained oscillator,... which continuously drives the separation bubble into states of expansion and contraction leading to shear layer flapping.' It is fundamentally distinct from the high-Reynolds-number instabilities in the shear layer itself which lead to transition, vortex growth, subsequent merging and 'shedding' from the rear of the bubble. There is, of course, an extensive literature on these latter instabilities and their control. A comprehensive review is provided by Greenblatt \& Wygnanski (2000) and it seems that it is control of these (essentially K-H) instabilities which appears to be the most effective in, inter alia, reducing the size of a separated region (see, for example, Siller \& Fernholz 1997). Whether the much lower-frequency modes identified by Hudy et al. can be controlled remains an open question.

Our present results are all satisfyingly consistent with Hudy et al.'s basic idea, although the situation is much less confusing because there is no turbulence. Since the reverse flow velocity is always large enough, once $R e$ exceeds $R e_{c a}$ over a sufficiently large region of the wake, a low-frequency global mode appears, whose character is exactly that suggested by Hudy et al. Its frequency, $f^{*}$, when computed using parameters $\left(\delta_{w}\right.$ and $\left.U_{a v}\right)$ at the location where the magnitude of the negative velocity is greatest, is in the range $0.006<f^{*}<0.043$ at conditions just beyond $R e_{c g}$, depending on the Reynolds number. For the high-Reynolds-number turbulent bubble studies mentioned above, the global mode frequency is essentially independent of $R e$, although defining the latter on the basis of an effective eddy viscosity gives values of the same order as those used in the present study. Note that using linear stability analysis for parallel flow is unlikely to be very useful for determining the frequency of the fully saturated global mode found in our NS computations, but it is significant that the latter give scaled frequencies similar to those found in fully turbulent studies.

What remains rather unclear, however, is the precise mechanism which sets the time scale of the global mode. Note first that all the laboratory and numerical experiments on higher-Reynolds-number turbulent bubbles in which a low-frequency mode has appeared have been conducted with fixed finite-distance upper boundaries (either stress-free or no-slip) so that, in the present context, the location of that boundary could be crucial in controlling the possible existence of the low-frequency mode. Although the argument in $\S 3$ which suggests that the period might increase like $H^{2} R e$ seems incorrect in that our NS data show periods varying significantly more rapidly, there is no doubt that the period increases with $R e$ and even more rapidly with $H$-indeed, the period does appear to scale with $H^{2} R e$. Whether this continues for even larger $H$ so that in an infinite domain the mode would effectively disappear (having an infinite period) is not known. Such a conclusion is only partially consistent with the implications of the stability calculations; all the NS computations give maximum negative velocities of around $35 \%$ (before the global mode sets in) and at this level, figure 10 shows that increasing the domain size leads to a reduction in 
$R e_{c a}$. Scaling the periods at the 'just critical' Reynolds number, $R e_{c a}$, in the same way as the data in figure 9 does not lead to any collapse. On the other hand, at a fixed $\lambda=1.35$, the period of the absolutely unstable mode at $R e_{c a}$ undoubtedly tends to rise with increasing $H$ (figure 11), which is consistent with the NS data. It is quite possible that the global mode period is set by conditions at a 'front' located at the upstream edge of the absolutely unstable region of the wake. Such fronts have been shown to exist at least for some classes of spatially developing wakes, and it is the frequency of the dominant mode at the front which determines that of the global mode (Pier \& Huerre 2001). This issue remains open and requires further work.

Nonetheless, we have shown unequivocally the appearance of a low-frequency global mode in a laminar separated bubble and its character - large-scale bubble expansion and contraction - has been identified. The results of the present NS computations are largely consistent with the implications of our new linear stability analysis for velocity profiles similar to those actually occurring in the wakes. If the domain height is too small (below about $H \simeq 4$ for the geometry studied here) the wake never contains a (low-frequency) absolutely unstable region, whatever the value of $R e$, and thus does not develop this global mode (although, of course, it becomes susceptible to higherfrequency $\mathrm{K}-\mathrm{H}$-type instabilities). If $H$ is large enough to allow such a mode, however, its period increases quite rapidly with increasing $H$ and $R e$. It is also concluded that very similar modes occur in high-Reynolds-number fully turbulent bubbles provided there is sufficient backflow velocity (and an upper boundary).

A more complete stability analysis, with inclusion of non-parallel and nonlinear effects, is required to identify the precise nature of the initial absolute instability and perhaps also to predict the frequency of the global mode. Whilst such an analysis may lead to more useful understanding of the initiation of this mode, it seems likely that only full NS solutions (via the laboratory or, as here, computations) can provide all the details of the character of the global mode itself in specific geometries.

The author wishes to thank his colleagues, Professor Sergei Chernyshenko, for many useful and instructive discussions, and Professor Neil Sandham, for provision of the Orr-Sommerfeld code and advice on its use. Thanks are also due to the referees, who made a number of helpful comments.

\section{REFERENCES}

Alam, M. \& Sandham, N. S. 2000 Direct numerical simulation of a 'short' laminar separation bubbles with turbulent reattachment. J. Fluid Mech. 410, 1-28.

Barkley, D., Gomes, M. G. M. \& Henderson, R. D. 2002 Three-dimensional instability in flow over a backward-facing step. J. Fluid Mech. 473, 167-190.

Barkley, D. \& Henderson, R. D. 1996 Three-dimensional Floquet stability analysis of the wake of a circular cylinder. J. Fluid Mech. 322, 215-241.

Buell, J. C. \& Huerre, P. 1988 Inflow/outflow boundary conditions and global dynamics of spatial mixing layers. Proc. NASA Ames/Stanford Centre for Turbulence Research Summer Programme, Rep. CTR-588, pp. 19-27.

Castro, I. P. 2002 Weakly stratified laminar flow past normal flat plates. J. Fluid Mech. 454, 21-46. 
Castro, I. P. \& Haque, A. 1987 The structure of a turbulnet shear layer bounding a separation region. J. Fluid Mech. 179, 439-468.

CHERnYSHEnKo, S. 1988 The asymptotic form of the stationary separated circumference of a body at high Reynolds number. Appl. Math. Mech 52, 746 (translated from Izv. Akad. Mekh. 52, 958-966.

Chernyshenko, S. 1993 Stratified Sadovskii flow in a channel. J. Fluid Mech. 250, 423431.

Chernyshenko, S. \& Castro, I. P. 1993 High-Reynolds-number asymptotics of the steady flow through a row of bluff bodies. J. Fluid Mech. 257, 421-449 (referred to herein as CA).

Chernyshenko, S. \& CAStro, I. P. 1996 High-Reynolds-number weakly stratified flow past an obstacle. J. Fluid Mech. 317, 155-178.

Cherry, N. J., Hillier, R. \& Latour, M. E. M. P. 1984 Unsteady measurements in a separated and reattaching flow. J. Fluid Mech. 144, 13-46.

FornberG, B. 1991 Steady incompressible flow past a row of circular cylinders. J. Fluid Mech. 225, $655-671$.

GajJar, J. \& Azzam, N. A. 2004 Numerical solution of the Navier-Stokes equations for the flow in a cylinder cascade. J. Fluid Mech. 520, 51-82.

GASTER, M. 1963 On stability of parallel flows and the behaviour of separation bubbles. PhD thesis, University of London.

GaSter, M. 1981 On transition to turbulence in boundary layers. Transition and Turbulence (ed. R. E. Meyer) pp. 95-112. Academic.

Greenblatt, D. \& Wygnanski, I. J. 2000 The control of flow separation by periodic excitation. Prog. Aero. Sci. 36, 487-545.

Hammond, D. A. \& Redekopp, L. G. 1998 Instability of separation bubbles. Eur. J. Mech. B/Fluids 17, $145-164$.

Heenan, A. F. \& Morrison, J. F. 1998 Passive control of pressure fluctuations generated by separated flow. AIAA J. 36, 1014.

Hudy, L. M., NAGuib, A. M. \& Humphries, W. M. 2003 Wall-pressure-array measurements beneath a separating/reattaching flow region. Phys. Fluids 15, 706-717.

Huerre, P. \& Monkewitz, P. A. 1985 Absolute and convective instabilities in free shear layers. J. Fluid Mech. 159, 151-168.

Huerre, P. \& Monkewitz, P. A. 1990 Local and global instabilities in spatially developing flows. Annu. Rev. Fluid Mech. 22, 473-537.

Hultgren, L. S. \& Aggarwal, A. K. 1987 Absolute instability of the Gaussian wake profile. Phys. Fluids 30, 3383-3387.

Ingham, D. B., TANG, T. \& Morton, B. R. 1990 Steady two-dimensional flow through a row of normal flat plates. J. Fluid Mech. 210, 281-302.

KIYA, M. \& SASAKI, K. 1985 Structure of large-scale votices and unsteady reverse flow in a reattaching zone of a turbulent separation bubble. $1985 \mathrm{~J}$. Fluid Mech. 154, 463-491.

LeONARD, B. P. \& MokhtaRI, S. 1990 Beyond first-order upwinding: the ultra-sharp alternative for non-oscillatory steady state simulation of convection. Intl J. Numer.Methods Engng 30, 729-766.

LOWERY, P. S. \& REYNOLDS, W. C. 1986 Numerical simulation of a spatially-developing, forced plane mixing layer. Rep. TF-68. Thermosciences Div., Dept of Mech. Engng, Stanford University.

Manhart, M. \& Friedrich, R. 2001 DNS of a turbulent boundary layer with separation. Proc. 2nd Conf. on Turbulence and Shear Flow Phenomena, KTH Stockholm, pp. 371-376.

MichalKe, A. 1965 On spatially growing disturbances in an inviscid shear layer J. Fluid Mech. 23, 521-544.

Natarajan, R., Fornberg, B. \& Acrivos, A. 1993 Flow past a row of flat plates at large Reynolds numbers. Proc. R. Soc. Lond. A441, 211-235.

NA, Y. \& MoIN, P. 1998 Direct numerical simulation of a separated turbulent boundary layer. J. Fluid Mech. 374, 379-405.

NiEw, M. F. 1993 The stability of the flow in a laminar separation bubble. PhD thesis, University of Cambridge.

Papageordiou, D. T. \& Smith, F. T. 1989 Linear instability of the wake behind a flat plate placed parallel to a uniform stream. J. Fluid Mech. 208, 67-89.

Patankar, S. V. 1980 Numerical Heat Transfer and Fluid Flows. McGraw-Hill. 
Pauley, L. L., Moin, P. \& Reynolds, W. C. 1990 The structure of two-dimensional separation. J. Fluid Mech. 220, 397-411.

Pier, B., Huerre, P., Chomaz, J.-M. \& Couairon, A. 1998 Steep nonlinear global modes in spatially developing media. Phys. Fluids 10, 2433-2437.

Pier, B. \& Huerre, P. 2001 Nonlinear self-sustained structures and fronts in spatially developing wake flows. J. Fluid Mech. 435, 145-174.

Siller, H. A. \& Fernholz, H. H. 1997 Control of separated flow downstream of a two-dimensional fence by low-frequency forcing. Appl. Sci. Res. 57, 309-318.

Smith, F. T. 1985 On large-scale eddy closure. J. Math. Phys. Sci. 19, 1-80. 